\title{
Primary hepatic neuroendocrine tumor: A case report
}

\author{
ASAHIRO MORISHITA ${ }^{1}$, HIROHITO YONEYAMA ${ }^{1}$, TAKAKO NOMURA ${ }^{1}$, TEPPEI SAKAMOTO ${ }^{1}$, \\ KOJI FUJITA ${ }^{1}$, JOJI TANI ${ }^{1}$, HISAAKI MIYOSHI ${ }^{1}$, REIJI HABA ${ }^{2}$ and TSUTOMU MASAKI ${ }^{1}$ \\ Departments of ${ }^{1}$ Gastroenterology and Neurology, and ${ }^{2}$ Diagnostic Pathology, \\ Faculty of Medicine, Kagawa University, Kagawa 761-0793, Japan
}

Received July 9, 2015; Accepted November 5, 2015

DOI: $10.3892 / \operatorname{mco} .2016 .822$

\begin{abstract}
We herein present a case of an 87-year-old patient with multiple liver tumors identified on abdominal ultrasound. The assessment performed on admission included physical examination, computed tomography (CT) during hepatic angiography and CT during arterial portography. The examination revealed contrast enhancement of a proportion of the liver tumors (20 $\mathrm{mm}$ maximum diameter) during the arterial phase and mild contrast washout of those tumors during the delayed phase. On contrast-enhanced magnetic resonance imaging using gadolinium ethoxybenzyl diethylenetriamine pentaacetic acid, certain liver tumors exhibited contrast enhancement during the early phase and contrast washout during the hepatocyte phase in both lobes. By contrast, no lesions were identified during positron emission tomography imaging of the liver. A liver biopsy was performed and immunohistochemical staining revealed enhanced expression of cytokeratin AE1/AE3, synaptophysin, chromogranin A and CD56 and no expression of hepatocyte antigen or CK7. The mindbomb E3 ubiquitin protein ligase-1 index was $2 \%$ in most of the tumor. The liver tumors were finally diagnosed as multiple intrahepatic metastases from a primary hepatic neuroendocrine tumor (PHNET). The patient underwent transarterial chemoembolisation with a combination of miriplatin (84 mg) mixed with gelatin sponge particles and lipiodol. To the best of our knowledge, this is the first report of PHNET in an patient aged $>85$ years.
\end{abstract}

\section{Introduction}

Neuroendocrine tumors (NETs) have an average annual incidence of 2 per 100,000 cases among all tumors of the gastrointestinal tract. NETs primarily arise in the

Correspondence to: Dr Asahiro Morishita, Department of Gastroenterology and Neurology, Faculty of Medicine, Kagawa University, 1750-1 Ikenobe, Miki-cho, Kida-gun, Kagawa 761-0793, Japan

E-mail: asahiro@med.kagawa-u.ac.jp

Key words: primary hepatic neuroendocrine tumor, transarterial chemoembolisation, miriplatin, multiple liver tumors, cytokeratin AE1/AE3, synaptophysin, chromogranin A, CD56 bronchopulmonary or gastrointestinal tracts (e.g., pancreas, ileum or appendix) and account for $\sim 70 \%$ of all NETs found in the body (1-3). However, NETs are frequently reported as metastatic liver tumors, although the liver itself is rarely described as the site of the primary tumor. Since Edmondson first reported a case of primary hepatic NET (PHNET) (4), $<100$ cases have been reported in the English-language literature $(3,5-9)$. The clinicopathological characteristics of PHNETs reveal that (i) they occur at a relatively young age (mean, 45 years), (ii) there is no known gender predominance, (iii) the majority of the cases are asymptomatic, (iv) the pathological diagnosis requires immunohistochemistry and (v) various therapeutic approaches may be attempted for PHNETs, such as hepatic lobectomy, systemic chemotherapy, transhepatic arterial chemoembolization (TACE), radiofrequency ablation and liver transplantation (10-12). To the best of our knowledge, there has been no report of PHNET with multiple liver metastases in a patient aged $>85$ years to date.

\section{Case report}

A 87-year-old man was referred to Kagawa University Hospital (Kagawa, Japan) with multiple liver tumors identified on abdominal ultrasound. The assessment performed on admission included physical examination, computed tomography (CT) during hepatic angiography and CT during arterial portography, and revealed multiple liver tumors (20 mm maximum diameter) (Fig. 1A). The liver tumors also exhibited hyperechogenicity and hypoechogenicity with acoustic shadows on ultrasonography (data not shown). On contrast-enhanced magnetic resonance imaging (MRI) using gadolinium ethoxybenzyl diethylenetriamine pentaacetic acid, certain tumors exhibited contrast enhancement during the early phase and contrast washout during the hepatocyte phase (Fig. 1B). By contrast, no lesions were identified during positron emission tomography (PET) imaging of the liver (data not shown). A liver biopsy was performed and immunohistochemical staining revealed enhanced expression of cytokeratin (CK) AE1/AE3, synaptophysin, chromogranin A and CD56, and no expression of hepatocyte antigen or CK7 (Fig. 2). No other primary lesion was detected and the patient was diagnosed with PHNETs. The mindbomb E3 ubiquitin protein ligase-1 index was $\sim 2 \%$ in most of the tumor. The patient underwent TACE with a combination of miriplatin (total dose of $84 \mathrm{mg}$ ) 

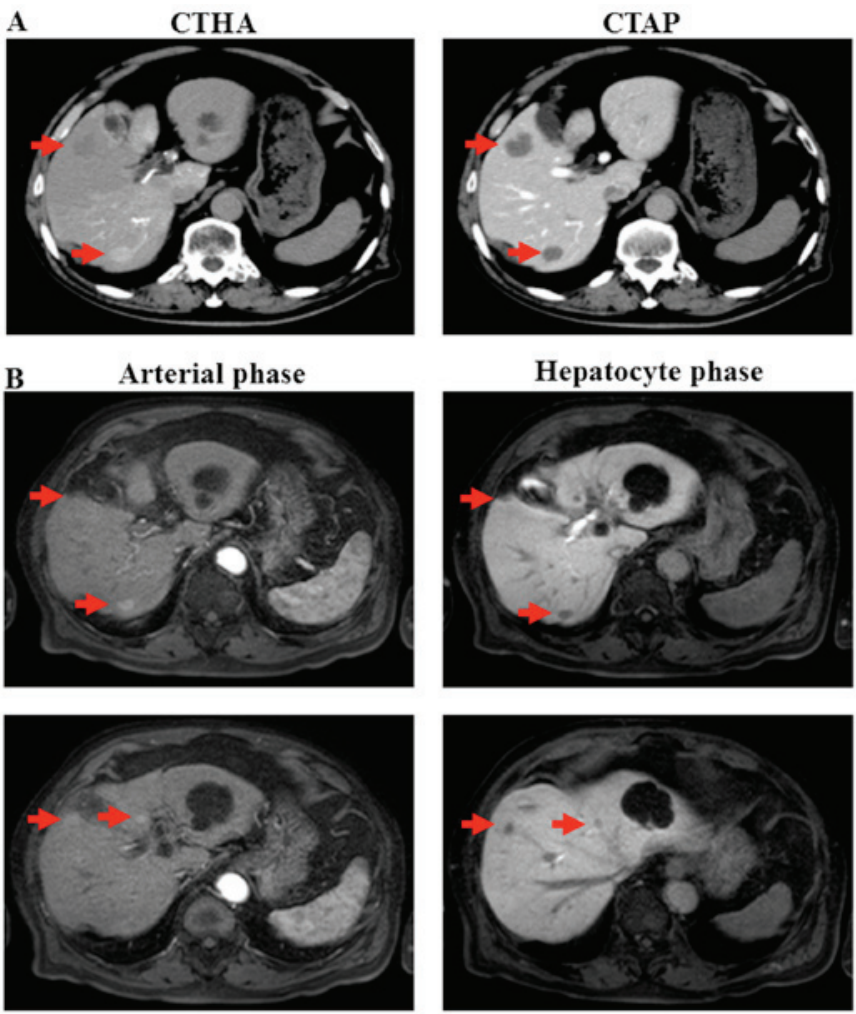

Figure 1. (A) Computed tomography (CT) images during hepatic angiography (CTHA) and arterial portography (CTAP). (B) Contrast-enhanced magnetic resonance imaging using gadolinium ethoxybenzyl diethylenetriamine pentaacetic acid during the arterial phase (left two panels) and the hepatocyte phase (right two panels). The tumors are indicated by the arrows.
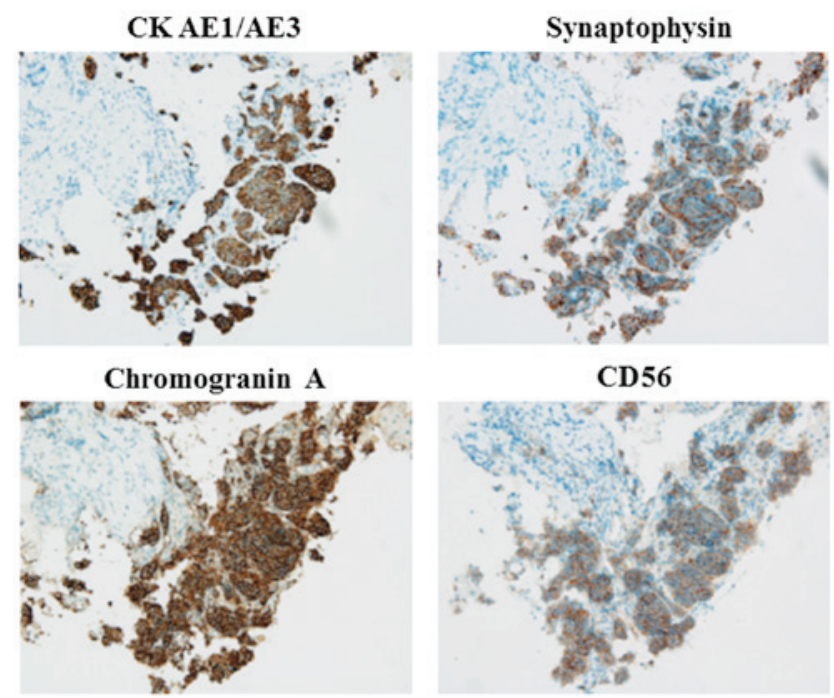

Hepatocyte antigen

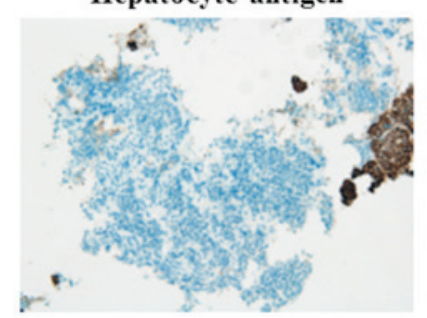

CK7

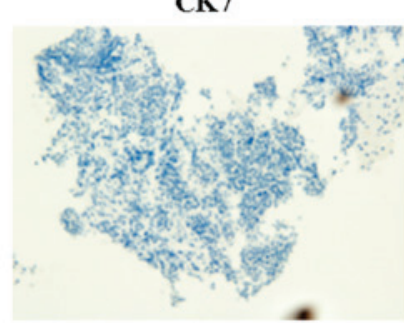

Figure 2. Immunohistochemistry was positive for the neuroendocrine tumor (NET) markers cytokeratin (CK) AE1/AE3, synaptophysin, chromogranin A and CD56 and negative for hepatocyte antigen and CK7 in primary hepatic NET tissues. mixed with gelatin sponge particles and lipiodol. The patient was treated using 3 courses of TACE, and partial response was identified during the follow-up examination, 21 months after the liver biopsy.

\section{Discussion}

NETs commonly develop in the gastrointestinal tract, pancreas and bronchopulmonary tract. In the majority of those cases, NETs detected in the liver have metastasized from different organs, and primary NETs originating in the liver are quite rare (13). PHNETs may be difficult to diagnose, even with pathological evidence, and must be differentiated from metastatic liver tumors. Therefore, clinical characteristics and imaging methods, including CT and MRI, are also crucial for definitively diagnosing PHNETs. In the present study, we diagnosed multiple liver tumors as PHNETs by histology and by applying various imaging modalities, such as CT, MRI and PET.

Non-specific symptoms, such as abdominal pain and distension, may be associated with the early stages of this disease. Additionally, several patients with gastrointestinal NETs suffer from carcinoid syndrome (14). This syndrome occurs in $<10 \%$ of patients with gastrointestinal NETs and is associated with liver metastasis. Of note, carcinoid syndrome is rarely observed in PHNET patients (15).

PHNETs primarily occur in patients between aged 40-50 years and are usually located in the right lobe of the liver (10). In the present study, the onset age of PHNET was 87 years and the tumors were located in both lobes of the liver. PHNETs may slowly metastasize to the other side of the hepatic lobe. To date, this is the first report of a PHNET patient aged $>85$ years.

Although the origin of PHNETs has not been elucidated, it has been hypothesized that NET cells may spread to the intrahepatic biliary tract and undergo malignant transformation, or that malignant stem cells may be transdifferentiated to NET cells (16). As PHNETs are rare, slow-growing and asymptomatic, early-stage diagnosis may be difficult. In the present study, a patient aged 87 years presented with multiple intrahepatic tumors that were diagnosed as PHNETs. Therefore, it is crucial to elucidate the etiology and mechanism underlying the development of these tumors.

Immunohistochemical analysis is the most effective method for the diagnosis of PHNETs. In our study, representative immunohistochemical markers, such as CK AE1/AE3, synaptophysin, chromogranin A and CD56, were positive (Fig. 2). Additionally, hepatocyte antigen and CK7, which are not expressed in NETs, were negative (Fig. 2). Sundin et al (17) reported that synaptophysin and chromogranin A were useful markers for a definitive diagnosis of PHNETs. Moreover, the quantification of chromogranin A in the plasma may be used for the follow-up evaluation of NETs (1). These reports suggest that immunohistochemical markers are powerful tools for diagnosing PHNETs.

Surgical treatment is the only curative method, with 5- and 10 -year survival rates of 78 and $59 \%$, respectively (18). The majority of the PHNET patients underwent surgical resection if surgery was indicated. In our case, multiple PHNETs were detected in both lobes of the liver, and surgical resection 
was not considered to be an option. Therefore, TACE was performed with cisplatin. Yao et al (19) reported that hepatic chemoembolization for NETs effectively improves the clinical symptoms and achieves tumor control. TACE may be the most effective therapy for PHNETs with intrahepatic metastasis.

Radiofrequency ablation (RFA) may be used to treat HCCs sized $<5 \mathrm{~cm}(20,21)$ when $<3$ tumors are present $(21)$. Our case had at least 4 tumors in both hepatic lobes (Fig. 1); thus, RFA was not indicated. However, RFA may be useful for treating unresectable PHNETs.

In conclusion, PHNETs are rare, particularly in elderly individuals. Immunohistochemistry is key to accurately diagnosing PHNETs. Surgery is the only curative option, but TACE and/or RFA may be considered as alternative approaches in the case of unresectable PHNETs.

\section{References}

1. Modlin IM, Kidd M, Latich I, Zikusoka MN and Shapiro MD: Current status of gastrointestinal carcinoids. Gastroenterology 128: 1717-1751, 2005.

2. Modlin IM, Sandor A, Tang LH, Kidd M and Zelterman D: A 40-year analysis of 265 gastric carcinoids. Am J Gastroenterol 92: 633-638, 1997.

3. Modlin IM, Shapiro MD and Kidd M: An analysis of rare carcinoid tumors: Clarifying these clinical conundrums. World J Surg 29: 92-101, 2005

4. Edmondson $\mathrm{H}$ : Tumor of the liver and intrahepatic bile duct. In: Atlas of Tumor Pathology, section 7, fascicle 25. Armed Forces Institute of Pathology, Washington, pp[105-109, 1958.

5. Kehagias D, Moulopoulos L, Smirniotis V, Pafiti A, Ispanopoulos S and Vlahos L: Imaging findings in primary carcinoid tumour of the liver with gastrin production. Br J Radiol 72: 207-209, 1999.

6. Lin CW, Lai CH, Hsu CC, Hsu CT, Hsieh PM, Hung KC and Chen YS: Primary hepatic carcinoid tumor: A case report and review of the literature. Cases J 2: 90, 2009.

7. Schwartz G, Colanta A, Gaetz H, Olichney J and Attiyeh F: Primary carcinoid tumors of the liver. World J Surg Oncol 6: 91, 2008.

8. Zhu H, Sun K, Ward SC, Schwartz M, Thung SN and Qin L: Primary hepatic signet ring cell neuroendocrine tumor: A case report with literature review. Semin Liver Dis 30: 422-428, 2010.
9. Mima K, Beppu T, Murata A, Otao R, Miyake K, Okabe H, Masuda T, Okabe K, Sugiyama S, Chikamoto A, et al: Primary neuroendocrine tumor in the liver treated by hepatectomy: Report of a case. Surg Today 41: 1655-1660, 2011.

10. Gurung A, Yoshida EM, Scudamore CH, Hashim A, Erb SR and Webber DL: Primary hepatic neuroendocrine tumour requiring live donor liver transplantation: Case report and concise review. Ann Hepatol 11: 715-720, 2012.

11. Yang K, Cheng YS, Yang JJ, Jiang X and Guo JX: Primary hepatic neuroendocrine tumor with multiple liver metastases: A case report with review of the literature. World J Gastroenterol 21: 3132-3138, 2015

12. Lamberts SW, Hofland LJ and Nobels FR: Neuroendocrine tumor markers. Front Neuroendocrinol 22: 309-339, 2001.

13. Yalav O, Ülkü A, Akçam TA, Demiryürek H and Doran F: Primary hepatic neuroendocrine tumor: Five cases with different preoperative diagnoses. Turk J Gastroenterol 23: 272-278, 2012.

14. Shetty PK, Baliga SV, Balaiah K and Gnana PS: Primary hepatic neuroendocrine tumor: An unusual cystic presentation. Indian J Pathol Microbiol 53: 760-762, 2010.

15. Mehta DC, Warner RR, Parnes I and Weiss M: An 18-year follow-up of primary hepatic carcinoid with carcinoid syndrome. J Clin Gastroenterol 23: 60-62, 1996.

16. Kaya G, Pasche C, Osterheld MC, Chaubert P and Fontolliet C: Primary neuroendocrine carcinoma of the liver: An autopsy case. Pathol Int 51: 874-878, 2001.

17. Sundin A, Eriksson B, Bergström M, Långström B, Oberg K and Orlefors H: PET in the diagnosis of neuroendocrine tumors. An NY Acad Sci 1014: 246-257, 2004.

18. Knox CD, Anderson CD, Lamps LW, Adkins RB and Pinson CW: Long-term survival after resection for primary hepatic carcinoid tumor. Ann Surg Oncol 10: 1171-1175, 2003.

19. Yao KA, Talamonti MS, Nemcek A, Angelos P, Chrisman H, Skarda J, Benson AB, Rao S and Joehl RJ: Indications and results of liver resection and hepatic chemoembolization for metastatic gastrointestinal neuroendocrine tumors. Surgery 130: 677-682; discussion 682-685, 2001.

20. Boonsirikamchai P, Loyer EM, Choi $\mathrm{H}$ and Charnsangavej $\mathrm{C}$ : Planning and follow-up after ablation of hepatic tumors: Imaging evaluation. Surg Oncol Clin N Am 20: 301-315, viii, 2011.

21. Gamblin TC, Christians K and Pappas SG: Radiofrequency ablation of neuroendocrine hepatic metastasis. Surg Oncol Clin N Am 20: 273-279, vii-viii, 2011. 